\title{
A Review of the Emotional Aspects of Neuropathic Pain: From Comorbidity to Co-Pathogenesis
}

\author{
Riccardo Torta $\cdot$ Valentina Ieraci $\cdot$ Francesca Zizzi
}

Received: September 19, 2017

(C) The Author(s) 2017. This article is an open access publication

\section{ABSTRACT}

Neuropathic pain is characterized by both sensory and affective disturbances, supporting the notion that pain and mood disorders share common pathogenetic mechanisms. Moreover, biological and neuroimaging data show that common brain areas are involved in the modulation of painful and emotional experiences. Improved understanding of the molecular mechanisms underlying the role of neuroinflammation in regulation of affective behavior in neuropathic pain states is important for the development of novel therapeutic strategies. Psychological issues must be considered a factor influencing treatment and outcome in patients with neuropathic pain.

Funding: Pfizer, Italy.

Keywords: Mood disorders; Molecular mechanisms; Neuroinflammation; Neuropathic pain; Therapeutic strategies

Enhanced content To view enhanced content for this article, go to http://www.medengine.com/Redeem/ 20DCF060253B492B.

R. Torta $\cdot$ V. Ieraci $(\bowtie) \cdot F$. Zizzi

Department of Neuroscience "Rita Levi Montalcini", University of Turin, Turin, Italy

e-mail: valentina.ieraci@unito.it

\section{INTRODUCTION}

Neuropathic pain is associated with a substantial economic burden for both individuals and society [1]. There is strong evidence available to suggest that patients with neuropathic pain experience worse health-related quality of life (QOL) than the general population [2-4], and a negative effect of neuropathic pain has also been reported in other health conditions $[5,6]$. Chronic pain with a neuropathic component has been shown to be associated with worse QOL, greater psychological distress, increased interference with sleep, and loss of more workdays than chronic pain without a neuropathic component [7].

Neuropathic pain is characterized by sensory symptoms such as gain or loss of somatosensory function, burning and evoked pain, as well as abnormal temporal summation [8]. Nerve injury models have shown sensory disturbances to arise from interactions between neurons, immune and immune-like glial cells, and other immune cell-derived inflammatory mediators, including cytokines and chemokines, ATP, histamine, bradykinin and prostaglandins; in this sense, neuropathic pain is a neuro-immune disorder [9]. This report discusses the relationships between mood disorders and neuropathic pain, with a focus on the pathogenetic mechanisms linking these two disorders, and reviews available treatment strategies. 


\section{Compliance with Ethics Guidelines}

This article is based on previously conducted studies and does not involve any new studies of human or animal subjects performed by any of the authors.

\section{MOOD DISORDERS IN PATIENTS WITH NEUROPATHIC PAIN}

Clinically, neuropathic pain is characterized by both sensory and affective disturbances, and this frequent comorbidity supports the notion that pain and mood disorders share some common pathogenetic mechanisms [10]. It is important, therefore, to treat an affective disorder that coexists with pain. The affective disturbances associated with pain include working memory dysfunction, impaired cognition, decreased appetite, depression, anhedonia, disruptions to sleep cycles, and impaired familial and social interactions [11]. The most common regions of the brain involved with pain in affective disturbances are the nucleus accumbens, the medial prefrontal cortex and the periaqueductal gray [12]. However, while available research highlights that there are some psychological variables common across different disorders, every pathology is characterized by some specific psychological aspects [10]. Neurological disorders associated with neuropathic pain are characterized by important associations between psychosocial factors and pain intensity.

The importance of emotional aspects of pain is particularly evident for depression and anxiety [10]. Depression is predictive for pain in many conditions, including chronic musculoskeletal pain, multiple sclerosis, post-stroke pain and Parkinson's disease [13-16]. Moreover, the presence of a neuropathic component correlates with higher values for depression and anxiety [17-19]. Depression can also be associated with alexithymia, with evidence suggesting that if these two factors are present together, they have a combined influence on the affective component of pain [20]. Anxiety is frequently present in patients with chronic pain conditions, and appears to be correlated with pain intensity [17, 21]. Neuropathic pain is associated with specific psychological factors: fear linked to the painful sensation and perceived danger associated with different activities may cause irritability and social withdrawal [10]. Importantly, an inadequate response of neuropathic pain to various drug therapies constitutes a substantial unmet need [1].

\section{PATHOGENETIC MECHANISMS OF PAIN AND AFFECTIVE DISTURBANCES}

Supraspinal neuroinflammation has a potential role in the development of affective disorders in patients with neuropathic pain conditions. Evidence suggests sickness behavior, cognitive impairment, depression and other neuropsychiatric disorders all have immune mechanisms associated with pro-inflammatory cytokines and chemokines [22]. There is growing evidence, particularly from preclinical models, that nerve injury leads to specific neuroinflammation in affective forebrain regions, with such injury responsible for these affective disturbances; nerve injury-evoked supraspinal neuroinflammation has been shown to underlie the development of affective disturbances by disrupting critical physiological processes [11].

\section{Peripheral Inflammation and Affective Disturbances}

Peripheral inflammation, or an immune challenge, is known to trigger a constellation of symptoms, including fatigue, pain, an altered sleep pattern, anorexia and affective disturbances, many of which represent adaptive responses considered to promote survival, and are collectively termed sickness behavior [23]. Clinically, major depressive disorder has a marked inflammatory component, with elevated blood levels of the pro-inflammatory cytokines (TNF and IL-6) [24]. Furthermore, depressed patients with painful neuropathy have higher TNF levels than patients with painful neuropathy who do not have depression; this observation supports the existence of 
a common inflammatory mechanism for neuropathic pain and mood disorders [24].

\section{Immune-to-Brain Signaling and Inflammatory Cytokines as Neuromodulators}

Current understanding of immune-to-brain signaling comprises three non-exclusive signaling pathways: neural transmission, humoral transmission and molecular transmission [11]. Supraspinal cytokines and chemokines are thought to act as neuromodulators within a cytokine network of neurons, microglia and astrocytes, acting to regulate cytokine production and cytokine receptor expression, and both amplifying and attenuating cytokine signals. Nerve injury-evoked supraspinal neuroinflammation appears to disturb physiological cytokine concentrations, causing a 'sub-inflammatory' response [11]. Critical regions for affective state regulation, such as the hippocampus, hypothalamus, amygdala, nucleus accumbens and frontal cortical regions, show particularly strong expression patterns for cytokine and chemokine receptors.

\section{Inflammatory Cytokines and Affective Disturbances in the Neuropathic Brain}

Specific anatomical regions of the hippocampus, medial prefrontal cortex (mPFC), and interconnected regions such as the striatum/ NAcc, ventral tegmental area (VTA), hypothalamus, amygdala and periaqueductal gray (PAG) are critical for the regulation of affective behaviors [25]. Peripheral neuropathy leads to increased expression of pro-inflammatory cytokines as well as increased glial activation within these brain structures, and emerging evidence suggests that neuroinflammation in these brain regions leads to affective disturbances [26]. In the MPFC, hippocampus and the ventral postero-lateral thalamus and brainstem structures, neuroinflammation induced by nerve injury has been linked with both allodynia and hyperalgesia [11]. Emerging evidence has led to the proposal of a model in which it is postulated that, after nerve injury, the transmission of peripheral inflammation to the brain via immune-to-brain signaling pathways in the interconnected hippocampal-mPFC circuitry leads to supraspinal neuroinflammation, and is critical to the development of depression, anhedonia, cognitive impairment and sleep disturbances [11]. It is thought that there is a glutamatergic modulation system involved, either directly via elevated glutamate levels, altered expression of NMDA and AMPA receptors and glutamate transporters, or indirectly through metabolites of the indoleamine 2,3-dioxygenase pathway [26]. Suppression of critical brain neurotrophic factors by cytokines may lead to reduced neurogenesis and neuroplasticity. TNF modulation of the dopaminergic systems has been shown to be associated with blunted reward, and midbrain glial activation may play a role in midbrain cell death as well as altered glucocorticoid expression associated with affective disturbances [11]. Further detailed anatomical examination along with improved understanding of the precise molecular mechanisms underlying the role of neuroinflammation in regulation of affective behavior in neuropathic pain states is likely to be important for the development of novel therapeutic strategies.

\section{ANTIDEPRESSANTS AND ANTICONVULSANTS FOR NEUROPATHIC PAIN}

The common biological background in many emotional and pain states may, at least in part, explain the frequent association between pain and a depressed mood, and biological and neuroimaging data show that common brain areas are involved in the modulation of both painful and emotional experiences [27]. Increased levels of pro-inflammatory cytokines have been found to be associated with both depressive disorders and pain, suggesting that activation of the neuroimmune system may be a factor underlying the high co-occurrence of depression, pain and other medical disorders [26].

Neuropathic pain is widely recognized as one of the most intractable pain conditions to 
manage, with clinical outcomes often unsatisfactory [7]. When treating chronic pain, the ultimate goal is to reduce, but not necessarily eliminate pain, but to achieve better QOL, by restoring normal function and reducing comorbidities such as depression, anxiety and sleep disorders [7].

The activity of antidepressants on pain shows two phases: the first is fast and acts on pain directly, and the second becomes apparent later, through mood normalization [27]. Of the psychoactive agents, tricyclic antidepressants and serotonin-norepinephrine reuptake inhibitors are the only classes shown in randomized controlled trials to be effective for pain, and are currently indicated as guideline-recommended first-line treatment for neuropathic pain therapy $[1,10]$. Tricyclic antidepressants have been shown to have both anxiolytic and analgesic effects at low doses, without antidepressant activity [10]. In contrast, even at low doses, the selective serotonin reuptake inhibitors and dual-acting serotonergic and noradrenergic antidepressants have antidepressant, anxiolytic and analgesic effects [10]. While conventional antipsychotic agents have been used regularly to treat pain in recent decades, the occurrence of adverse effects, combined with the lack of specificity of their analgesic action, are important limitations; the atypical antipsychotics have better tolerability [10]. Antidepressants have been shown to reduce chronic pain in both depressed and non-depressed patients, suggesting that they have independent analgesic mechanisms, which combined with their ability to treat comorbid depression and pain-related sleep issues, suggest these agents may be of particular utility [28].

The role of adult hippocampal neurogenesis in emotions remains to be fully elucidated, although several studies have suggested that this process may be involved in both cognitive and emotional function and that it is deregulated in neuropsychiatric disorders such as major depression. Several psychoactive drugs, including antidepressants and anticonvulsants, can modulate neurogenesis in adults [29].
Growing evidence suggests that gabapentin and pregabalin are able to produce concentration-dependent increases in the numbers of newborn mature and immature neurons, along with a parallel decrease in the number of undifferentiated precursor cells [29]. In stressed adult mice, anticonvulsant administration has been shown to prevent the appearance of depression-like behaviors induced by chronic restraint stress and to promote hippocampal neurogenesis, although the clinical relevance of this finding, if any, remains to be clarified [29]. It is likely that several disorders, including anxiety, epilepsy, pain, fibromyalgia and sleep interference, may share neuronal hyperactivity in various brain circuits, and available data suggest the existence of novel molecular pathways that may be potential targets for new therapeutics [29].

The combination of antidepressants and anticonvulsants (i.e. gabapentin and duloxetine, pregabalin and duloxetine) may provide increased efficacy over monotherapy, but further studies of combination therapies are needed [28, 30, 31]. The combination of duloxetine and gabapentinoids was generally well tolerated, with an adverse event profile and rate similar to those of duloxetine monotherapy, with the exception of significantly less insomnia occurring in the combination treatment group [30]. The combination of pregabalin and duloxetine was also generally well tolerated; however, moderate to severe drowsiness was more frequent during combination therapy [31]. Patients with neuropathic pain often fail to respond adequately to pharmacologic treatments and their pain is therefore termed refractory [28]. An integrated psychopharmacological and psychological approach is necessary for treatment in some patients [10]. Psychoeducational interventions can improve treatment adherence, while cognitive behavioral intervention may be more useful in modifying distorted beliefs on pain. When focusing on the patient's life experiences, brief psychodynamic psychotherapy interventions can also be useful. 


\section{PSYCHOLOGICAL FACTORS AS PREDICTORS OF PAIN PERCEPTION}

Perceived pain intensity is not proportional to the type or extent of tissue damage, but depends on the interaction between cognitive, emotional, socio-cultural and physical factors $[10,32]$, and can be strongly influenced by stress, anxiety and depression [33]. Conversely, it is important that the role of pain on emotional and cognitive performance is established. Early assessment for coexisting psychiatric comorbidity using appropriate methods may help achieve early referral to a psychologist or a psychiatrist [28].

It is also important to highlight that in several neurological conditions, catastrophism is a factor that is associated with or predicts pain. Neurological conditions where catastrophism has affected pain include multiple sclerosis, lumbar or musculoskeletal pain, migraine, postherpetic neuralgia, cerebral palsy, the sequelae of severe traumatic brain injuries, as well as neuropathic pain following surgical procedures, neuropathic pain due to HIV and phantom limb pain [10].

\section{CONCLUSION}

Neuropathic pain is widely recognized as one of the most intractable pain conditions to manage, with clinical outcomes often unsatisfactory. Psychological factors such as depression and anxiety must be taken into consideration as factors that may influence treatment and outcome. Moreover, when treating chronic pain, it must be remembered that the ultimate goal is not simply to reduce pain, but to achieve better QOL, by restoring normal function and reducing comorbidities such as depression, anxiety and sleep disorders.

\section{ACKNOWLEDGEMENTS}

This supplement has been sponsored by Pfizer, Italy. The article processing charges for this publication were also funded by Pfizer, Italy.

The authors thank Marie Cheeseman who provided English editing of the manuscript on behalf of Springer Healthcare Communications. This medical writing assistance was funded by Pfizer, Italy. All named authors meet the International Committee of Medical Journal Editors (ICMJE) criteria for authorship for this manuscript, take responsibility for the integrity of the work as a whole and have given final approval to the version to be published.

The authors equally contributed to the preparation of the manuscript.

Disclosures. Riccardo Torta reports receiving honorarium from Pfizer. Valentina Ieraci and Francesca Zizzi have nothing to disclose.

Compliance with Ethics Guidelines. This article is based on previously conducted studies and does not involve any new studies of human or animal subjects performed by any of the authors.

Data Availability. Data sharing is not applicable to this article as no datasets were generated or analyzed during the current study.

Open Access. This article is distributed under the terms of the Creative Commons Attribution-NonCommercial 4.0 International License (http://creativecommons.org/licenses/ by-nc/4.0/), which permits any noncommercial use, distribution, and reproduction in any medium, provided you give appropriate credit to the original author(s) and the source, provide a link to the Creative Commons license, and indicate if changes were made. 


\section{REFERENCES}

1. Finnerup NB, Attal N, Haroutounian S, et al. Pharmacotherapy for neuropathic pain in adults: a systematic review and meta-analysis. Lancet Neurol. 2015;14(2):162-73.

2. Doth AH, Hansson PT, Jensen MP, Taylor RS. The burden of neuropathic pain: a systematic review and meta-analysis of health utilities. Pain. 2010;149(2):338-44.

3. Meyer-Rosberg K, Burckhardt CS, Huizar K, Kvarnström A, Nordfors LO, Kristofferson A. A comparison of the SF-36 and Nottingham Health Profile in patients with chronic neuropathic pain. Eur J Pain. 2001;5(4):391-403.

4. Smith BH, Torrance N, Bennett MI, Lee AJ. Health and quality of life associated with chronic pain of predominantly neuropathic origin in the community. Clin J Pain. 2007;23(2):143-9.

5. Crane AM, Levitt RC, Felix ER, Sarantopoulos KD, McClellan AL, Galor A. Patients with more severe symptoms of neuropathic ocular pain report more frequent and severe chronic overlapping pain conditions and psychiatric disease. Br J Ophthalmol. 2017;101(2):227-31.

6. Dermanovic Dobrota V, Hrabac P, Skegro D, et al. The impact of neuropathic pain and other comorbidities on the quality of life in patients with diabetes. Health Qual Life Outcomes. 2014;12(1):171.

7. Inoue $\mathrm{S}$, Taguchi $\mathrm{T}$, Yamashita $\mathrm{T}$, Nakamura $\mathrm{M}$, Ushida $\mathrm{T}$. The prevalence and impact of chronic neuropathic pain on daily and social life: a nationwide study in a Japanese population. Eur J Pain. 2017;21(4):727-37.

8. Gierthmuhlen J, Baron R. Neuropathic pain. Semin Neurol. 2016;36(5):462-8.

9. Martini R, Willison H. Neuroinflammation in the peripheral nerve: cause, modulator, or bystander in peripheral neuropathies? Glia. 2016;64(4):475-86.

10. Aloisi AM, Berlincioni V, Torta R, et al. The role of gender, psycho-social factors and anthropological-cultural dimensions on pain in neurorehabilitation. Evidence and recommendations from the Italian Consensus Conference on Pain in Neurorehabilitation. Eur J Phys Rehabil Med. 2016;52(5):730-40.

11. Fiore NT, Austin PJ. Are the emergence of affective disturbances in neuropathic pain states contingent on supraspinal neuroinflammation? Brain Behav Immun. 2016;56:397-411.
12. Descalzi G, Mitsi V, Purushothaman I, et al. Neuropathic pain promotes adaptive changes in gene expression in brain networks involved in stress and depression. Sci Signal. 2017;10(471).

13. Brochet B, Deloire MS, Ouallet JC, et al. Pain and quality of life in the early stages after multiple sclerosis diagnosis: a 2-year longitudinal study. Clin J Pain. 2009;25(3):211-7.

14. O'Donnell MJ, Diener H-C, Sacco RL, Panju AA, Vinisko R, Yusuf S. Chronic pain syndromes after ischemic stroke: PRoFESS Trial. Stroke. 2013;44(5):1238-43.

15. Wasserman RA, Brummett CM, Goesling J, Tsodikov A, Hassett AL. Characteristics of chronic pain patients who take opioids and persistently report high pain intensity. Reg Anesth Pain Med. 2014;39(1):13-7.

16. Wen H-B, Zhang Z-X, Wang H, et al. Epidemiology and clinical phenomenology for Parkinson's disease with pain and fatigue. Parkinsonism Relat Disord. 2012;18(Suppl 1):S222-5.

17. Radat F, Margot-Duclot A, Attal N. Psychiatric co-morbidities in patients with chronic peripheral neuropathic pain: a multicentre cohort study. Eur J Pain. 2013;17(10):1547-57.

18. Shaygan M, Böger A, Kröner-Herwig B. Clinical features of chronic pain with neuropathic characteristics: a symptom-based assessment using the pain DETECT questionnaire. Eur J Pain. 2013;17(10):1529-38.

19. Uher T, Bob P. Neuropathic pain, depressive symptoms, and C-reactive protein in sciatica patients. Int J Neurosci. 2013;123(3):204-8.

20. Hosoi M, Molton IR, Jensen MP, et al. Relationships among alexithymia and pain intensity, pain interference, and vitality in persons with neuromuscular disease: considering the effect of negative affectivity. Pain. 2010;149(2):273-7.

21. McWilliams LA, Goodwin RD, Cox BJ. Depression and anxiety associated with three pain conditions: results from a nationally representative sample. Pain. 2004;111(1-2):77-83.

22. Stuart MJ, Baune BT. Chemokines and chemokine receptors in mood disorders, schizophrenia, and cognitive impairment: a systematic review of biomarker studies. Neurosci Biobehav Rev. 2014;42:93-115.

23. McCusker RH, Kelley KW. Immune-neural connections: how the immune system's response to infectious agents influences behavior. J Exp Biol. 2013;216(Pt 1):84-98. 
24. Walker AK, Kavelaars A, Heijnen CJ, Dantzer R. Neuroinflammation and comorbidity of pain and depression. Pharmacol Rev. 2014;66(1):80-101.

25. Drevets WC, Price JL, Furey ML. Brain structural and functional abnormalities in mood disorders: implications for neurocircuitry models of depression. Brain Struct Funct. 2008;213(1-2):93-118.

26. Benatti C, Blom JM, Rigillo G, et al. Disease-induced neuroinflammation and depression. CNS Neurol Disord Drug Targets. 2016;15(4):414-33.

27. Torta RG, Ieraci V. Depressive disorders and pain: a joint model of diagnosis and treatment. J Pain Relief. 2013;S2:003.

28. Gilron I, Baron R, Jensen T. Neuropathic pain: principles of diagnosis and treatment. Mayo Clin Proc. 2015;90(4):532-45.

29. Valente MM, Bortolotto V, Cuccurazzu B, et al. $\alpha 2 \delta$ ligands act as positive modulators of adult hippocampal neurogenesis and prevent depression-like behavior induced by chronic restraint stress. Mol Pharmacol. 2012;82(2):271-80.
30. Tanenberg RJ, Irving GA, Risser RC, et al. Duloxetine, pregabalin, and duloxetine plus gabapentin for diabetic peripheral neuropathic pain management in patients with inadequate pain response to gabapentin: an open-label, randomized, noninferiority comparison. Mayo Clin Proc. 2011; 86(7):615-26.

31. Gilron I, Chaparro LE, Tu D, et al. Combination of pregabalin with duloxetine for fibromyalgia: a randomized controlled trial. Pain. 2016;157(7): 1532-40.

32. Torta RG, Munari J. Symptom cluster: depression and pain. Surg Oncol. 2010;19(3):155-9.

33. Castelnuovo G, Giusti EM, Manzoni GM, et al. Psychological considerations in the assessment and treatment of pain in neurorehabilitation and psychological factors predictive of therapeutic response: evidence and recommendations from the Italian Consensus Conference on Pain in Neurorehabilitation. Front Psychol. 2016;7:468. 